\title{
DYNAMIC COUPLING BETWEEN TOP-END VESSEL SWAY AND SLENDER RISER VIV IN DEEP WATER
}

\author{
Weimin Chen \\ Key Laboratory for Mechanics in Fluid Solid \\ Coupling System, Institute of Mechanics, \\ Chinese Academy of Sciences \\ Beijing , 100190, China
}

\author{
Shuangxi Guo \\ Key Laboratory for Mechanics in Fluid Solid \\ Coupling System, Institute of Mechanics , \\ Chinese Academy of Sciences \\ Beijing , 100190, China
}

\author{
Min Li \\ School of Aeronautics Sciences and Engineering, \\ Beijing University of Aeronautics and Astronautics \\ Beijing 100191, China
}

\author{
Kuan Gan \\ School of Aeronautics Sciences and Engineering, \\ Beijing University of Aeronautics and Astronautics \\ Beijing 100191, China
}

\begin{abstract}
The impacts of top-end motion on the riser undergoing vortex-induced vibration (VIV) are explored in this study, because the dynamic coupling between moving top-end vessel and submarine riser becomes more remarkable for a floating platform in deeper water due to the larger top-end motion amplitude, compared with the fixed platform in shallow water. A coupled hydrodynamic force approach, involving the vortexinduced lift force along with the fluid drag force, is developed. The dynamic responses of the system including a floating topend and a riser experiencing VIV are examined by means of finite element simulations. The effects of amplitude and frequency of top-end vessel sway on riser VIV are examined. Our numerical results show that the riser displacement becomes several times larger than the displacement for the case without top-end motion. Moreover, the nonlinear response amplification is observed, and the nonlinear amplification gets more pronounced as the number of mode order dropping, while the amplification factor just slightly changes with the increase of sway amplitude.
\end{abstract}

\section{INTRODUCTION}

Submarine riser of deep water platform has been becoming longer as the oil and gas exploration extending toward deeper ocean. Because the aspect ratio (the ratio of riser length to diameter) of deep water riser, connecting top vessel and sea bed, is getting large, i.e. to a magnitude order of $10^{2}$ or
$10^{3}$, structural modal frequencies are often low and dense. And, the fluid field, such as current speed and direction, are no longer uniformly distributes along riser span. Therefore more complicated phenomena, e.g. multi-mode vibration and widerband random vibration, of the vortex-induced vibration of long flexible risers are frequently observed (Chen et al.,2006; Heurtier et al., 2001; Lou et al., 2010; Stansberg et al., 2002; Tahara and Kim, 2003). On the other hand, compared with fixed platform in shallow water, floating platform in deep water has more significant motion amplitude and thus the coupling between top-end vessel and submarine riser appears to be more remarkable. Moreover, new issues such as additional lock-in region, parametric excitation and nonlinear amplification, due to the coupling effect are introduced (Garrett, 2005; Wang and Ling, 1998). It is reported that the tension fluctuation due to top-end heave may cause a riser VIV involving higher-order modes and increasing dynamic response, e.g. respectively $10 \%$ and $20 \sim 100 \%$ higher riser displacement and shear stress than the case without vessel motion (Wang and Ling, 1998). Rampazzo et al. (2008) pointed out the necessity of doing coupled analysis for a system involving a flexible SCR and a moving top-end (DP-Tugboat) by numerical simulations.

Among the researches on the dynamic coupling between top-end vessel and marine riser, most of them focus on top-end vessel dynamic response. Generally, the methods of those researches can be classified into two kinds: the quasi-static method (Ormberg et al., 1997; Kim et al., 2001; Spanos et al., 2005; Wichers et al., 2001) and the coupled method (Bosman 
and Hooker, 1999; Chen et al.,2006, Li et al 2010; Tahara and Kim, 2003). In the quasi-static method, riser is simplified as a spring with lumped mass, only the hydrostatic restoring force of riser exerting upon the top-end vessel is considered. Spanos et al. (2005) studied the influence of riser stiffness on the overall dynamic response of a SPAR platform by using a simplified model, in which the top-end vessel mass was concentrated at the gravity center and a horizontal spring was used to simulate the interaction between riser and vessel. Ormberg et al. (1997), Kim et al. (2001) and Wichers et al. (2001) investigated the interactions of floating top-end and riser (or mooring system) of deep-water platforms so as to compare the coupled approach in time-domain and the quasi-static approach. Their results show that the capability of mooring system bearing external loads may be underestimated by using the quasi-static approach.

In the coupled method, submarine riser and its hydrodynamic force exerted by ambient ocean current or wave are mostly simplified (Lee and Wang, 2000; Li et al., 2010; Tahara and Kim, 2008), e.g. the Morison formula is employed to model hydrodynamic force. The primary concern of previous researches is dynamic responses of riser rather than riser VIV. Lee and Wang (2000) used a linear tensional string to simulate tension leg and analyzed the leg dynamic response as the topend vessel experiencing periodical surge. His results showed that there is a leg vibration mode similar with the platform while the vibration amplitude changes with wave period. Tahara et al. (2008) employed the empirical formula of the Young's modulus suggested by Bosman and Hooker (1999) to examine the mooring system response of a SPAR undergoing heave. By comparing his result with that of a linearly elastic mooring system, he found a remarkable difference between the two results.

In addition, it is worthwhile to mention that the dynamic coupling mechanisms, between top vessel and submarine riser, due to different vessel motions are essentially different. Taking vessel heave as an example, it introduces a fluctuating tension of riser, which presents a periodically varying structural property, thus, the consequent may be the parametric excitation of riser. Sometimes, the oscillatory vertical motion imposed to the top of risers may result in a significant vortex-self induced vibration (Pereira et al., 2013). Whereas, if we consider the horizontal motions, i.e. sway or surge of vessel, the transverse vibration of top-end will propagate along riser. This transverse vibration may directly interact with riser VIV. Even, the vibration might be amplified during its propagation along riser. This introduces quite a different issue from the parametric excitation due to top-end heave. In this paper, only the dynamic interaction between top-end vessel sway and riser VIV are addressed.

First, we develop a hydrodynamic approach to model the vortex-induced lift force which essentially depends on structure motion. Then the dynamic response of the integrated system including top-end vessel sway and riser VIV are examined by means of finite element simulations. The effects of top-end sway amplitude and frequency on riser response displacement as well as the vibration propagation are examined so as to have a deeper insight into the interaction between top-end vessel sway and riser VIV.

\section{NUMERICAL MODEL OF DYNAMIC RESPONSE ANALYSIS FOR INTEGRATED SYSTEM}

\subsection{Structure Model}

The integrated system including the top-vessel and riser is shown in Fig.1. In Fig. 1a, the origin point of the coordinate system is located at the bottom end of the riser (fixed to the sea bed). The flow $U$ directs along the axis $y$. The sway motion of top vessel is $b(t)=B e^{-i \omega_{0} t}$, where $B$ and $\omega_{0}$ are respectively the amplitude and frequency of the sway. In the finite element model (shown in Fig.1b), the vessel and the riser respectively consist of 3D cubic solid and 1D Euler beam elements. The rotational motions around the axis $x, y$, and $z$ of all those grids of the top-end are constrained during the numerical simulations so as to avoid a probable singularity introduced by the extremely large mass of the top-end. Additionally, the multi-point constrain (MPC) is used at the joint grid connecting the top-end vessel and the riser, where different elements meet together, so that the constrains can be exerted smoothly upon different freedom degrees.

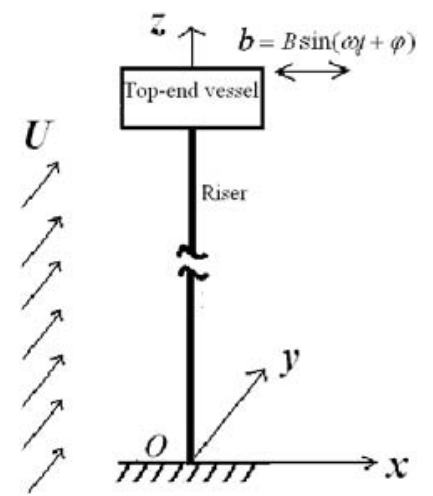

1a The platform-riser system and current distribution

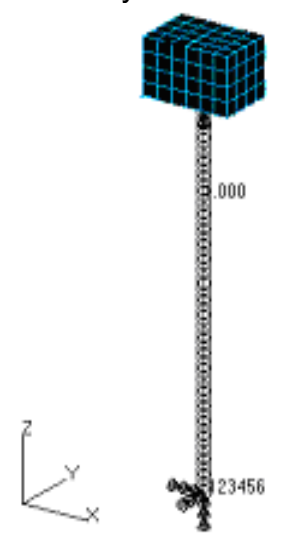

1b The finite element model of platform-riser system Figure 1 The platform-riser system sketch 

written as

The governing equation of the riser dynamics can be $E I \frac{\partial^{4} x(z, t)}{\partial z^{4}}-T \frac{\partial^{2} x(z, t)}{\partial z^{2}}+c \frac{\partial x(z, t)}{\partial t}+m \frac{\partial^{2} x(z, t)}{\partial t^{2}}=F(z, t)$

where $E I$ is the bending stiffness, $T$ is the top tension, $C$ is the structural damping, $m$ is the structural mass per unit length. $F(z)$ is the hydrodynamic force, of which the expression will be presented in next section. The boundary conditions at two ends of the riser are

$$
\begin{array}{ll}
x(0, t)=b(t) & x(L, t)=0 \\
\partial^{2} x(0, t) / \partial z^{2}=0
\end{array} \text { and } \quad \begin{aligned}
& \partial^{2} x(L, t) / \partial z^{2}=0
\end{aligned}
$$

\subsection{Hydrodynamic Force Model}

The hydrodynamic force $F(z)$ in Eq.(1) consists of two parts, i.e. the vortex-induced lift force $F_{L}$ and the fluid drag force $F_{D}$. The fluid drag force $F_{D}$ can be expressed by the Morison equation as

$F_{D}=\frac{1}{2} C_{D} \rho_{f} D(U-\dot{x})|U-\dot{x}|+\frac{1}{4} C_{a} \pi D^{2} \rho_{f}(\dot{U}-\ddot{x})+\frac{1}{4} \pi D^{2} \rho_{f} \dot{U}$ where $\rho_{f}$ and $U$ are the fluid density and velocity respectively. $D$ is the riser's outer diameter. $C_{D}$ and $C_{a}$ are the coefficients of drag forces and added mass respectively, of which the values are $C_{a}=1.0$ and $C_{D}=1.1$ for a flexible riser with large aspect ratio.

VIV has always been a challenging issue concerning the interaction between fluid and structural dynamics. It exhibits some interesting, and even unexplained until now, traits like self-excitation, self-limitation of response amplitude, a variety of vortex-shedding modes, multi-mode or wide-band random vibration. Initially, vortex-induced lift force per unit length of riser is somewhat similar with the Morison equation and written as

$$
F_{L}=\frac{1}{2} \rho_{f} U^{2} C_{L} D
$$

where the lift coefficient $C_{L}$ is a constant value. With recently increasing amount of experimental observations along with CFD simulations, deeper understandings of VIV were reported. New approaches of hydrodynamic force during lockin are proposed, which are more accurate and reasonable because of consideration of coupling between structural and fluid dynamics (Gopalfrishnan, 1993; Vandiver, 2002; Chen, 2012).

Sarpkaya (2004) experimentally measured the Fourier average of hydrodynamic force over many cycles of vibration. He decomposed the lift force into two parts, the drag part and the inertia part, which are respectively related to velocity and acceleration of a moving cylinder. He pointed out that for practical Reynolds number, the nonlinear expression in manners of structural motion is able to capture hydrodynamic feature better than the linear expression. Gopalfrishnan (1993) and Govardhan and Williamson (2004) implemented considerable numbers of VIV experiments and presented the lift coefficient versus structural motion. Vandiver (2002) suggested that a piecewise parabola function of structural amplitude could be used for the industrial model of lift force to calculate the riser displacement by using the wake oscillator model. Based on above studies, we suggest that lift coefficient $C_{L}$ depend on structure motion rather than merely a constant value.

A third-order polynomial of the structure velocity is used to model the lift force so that the nonlinear interaction between structural and fluid dynamics is taken into our account, i.e.

$$
\begin{aligned}
F_{L}(x) & =\frac{1}{2} \rho U^{2} D\left(C_{L 0} \sin (\omega t)+C_{1} \dot{x}(z, t)+C_{2} \dot{x}^{2}(z, t)+C_{3} \dot{x}^{3}(z, t)\right) \\
& =p_{f} C_{L}(\dot{x}(z, t))
\end{aligned}
$$

where $\quad p_{f}=\frac{1}{2} \rho U^{2} D$ and the lift coefficient is $C_{L}(\dot{y}(z, t))=C_{L 0} \sin (\omega t)+C_{1} \dot{x}(z, t)+C_{2} \dot{x}^{2}(z, t)+C_{3} \dot{x}^{3}(z, t)$. The values of the coefficients $C_{L 0}, C_{1}, C_{2}$ and $C_{3}$ can be derived by fitting experimental data. Among all VIV experiments, the results got in situations, of cylinder freely vibrating or flexible cable (with large aspect ratio) rather than forced vibration or rigid body, are strongly recommended, e.g. experiments of Gopalkrishnan (1993), Trim et al. (2005) and Chaplin et al. (2005). Chen et al. (2012) gave an approach to calculate the coefficients' values by fitting experimental data.

Observing Eq. (4), we may say it can capture, to some extent, the features of VIV. 1) The feature of self-excitation. In Eq. (4), the excitation is represented by the first term $p_{f} C_{L 0} \sin (\omega t)$, a sinusoidal excitation force, together with the second term, $p_{f} C_{1} \dot{x}(z, t)$, which increases as response increasing ( $C_{1}$ is required to be positive). 2) The feature of self-limitation. One of unique traits of VIV is that structural response never rises infinitely, but begins to drop when response amplitude reaches to a certain number, such as $\bar{y}_{\max }=1.5$ or 2.0. This feature, called self-limitation, is represented by the nonlinear terms with higher orders in Eq.(7), $\rho_{f} C_{2} \dot{x}^{2}(z, t)$ or $\rho_{f} C_{3} \dot{x}^{3}(z, t)$ (at least one of the coefficients $C_{2}$ and $C_{3}$ is negative). 3) Axially varying distribution of lift force along riser. For case of a rigid cylinder, the vortexinduced lift force uniformly distributes along riser. But for case of a flexible slender riser, the coherence may decrease due to the non-uniform distributions of lift force as well structural motion. In the present model, the lift force is non-uniform because of the axially-varying structural motion. Therefore, the span coherence of flexible riser is automatically captured.

\subsection{Validation against Experimental Results}

To validate the proposed model, the numerical results are compared to experimental results (see Fig. 2), i.e. a rigid cylinder undergoing uniform flow by Khalak and Williamson (1999), the flexible cylinders respectively undergoing a stepped 
flow by Chaplin et al. (2005) and a sheared flow by Trim et al. (2005).

In the numerical simulations, the hydrodynamic coefficients are set to be $C_{A}=1.0, C_{d}=1.1$ which were determined based on the corresponding experiments (Sarpkaya 2004). Regarding the lift force for a rigid cylinder is generally higher than a flexible cylinder (Liao and Vandiver, 2002; Chen et al., 2012), we set the lift coefficients to be $C_{L 0}=0.50$, $C_{L 1}=1.82, C_{L 2}=-1.29$, and $C_{L 3}=-0.71$ for the rigid cylinder. And $C_{L 0}=0.22, C_{L 1}=1.62, C_{L 2}=-2.31$ and $C_{L 3}=+0.75$ for the flexible cylinders. Figure 2 indicates that the calculated amplitudes have satisfied agreements with experimental results in different flow fields.

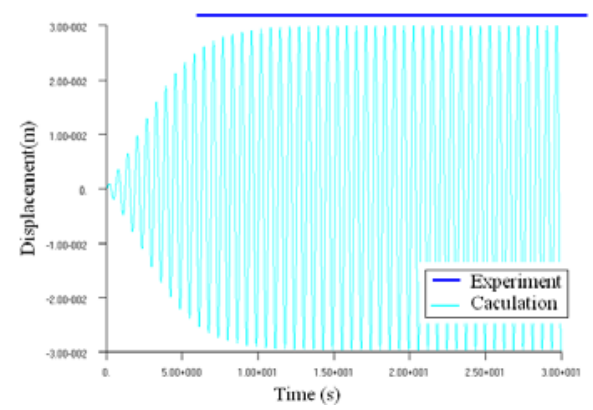

2a Rigid cylinder in uniform flow

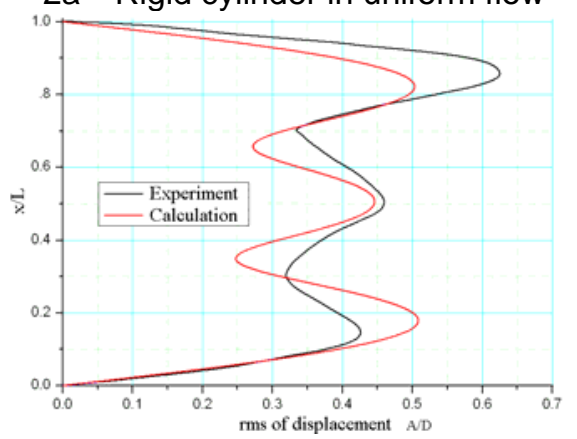

2b Flexible cylinder in stepped flow

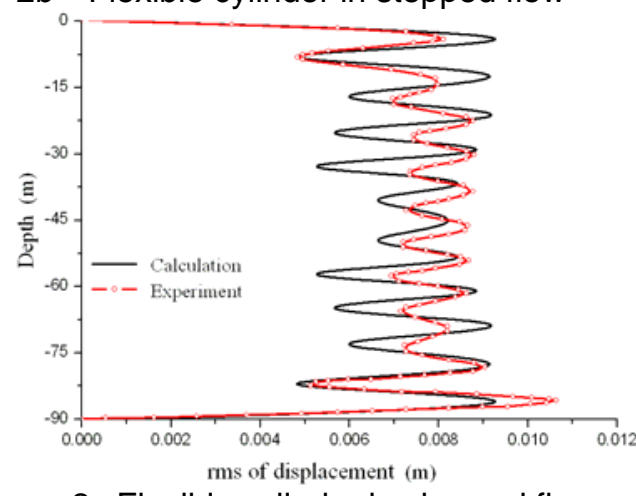

2c Flexible cylinder in sheared flow

Figure 2 Comparisons of VIV response between the presented numerical simulation and the existing experimental results of (a) rigid cylinder in uniform flow; (b) flexible cylinder in stepped flow; and (c) flexible cylinder in sheared flow.

\section{EFFECTS OF TOP-END VESSEL SWAY ON RISER VIV}

By combining the presented hydrodynamic model with the structure model, we can run the dynamic response calculation of the integrated system (shown in Fig.1) by using FEM code (Chen et al., 2012). In order to explore the impacts of top-end vessel sway on riser VIV, we analyze the riser response displacement and vibration wave propagation along riser at different sway amplitudes or frequencies.

The structural parameters of the riser are as follows: the outer and inner diameters are respectively $D=0.500 \mathrm{~m}$ and $d=0.445 \mathrm{~m}$. The riser length is $500 \mathrm{~m}$, and the material density is $\rho_{s}=7.8 \times 10^{3} \quad\left(\mathrm{~kg} / \mathrm{m}^{3}\right)$. The bending stiffness is $E I=3.8 \times 10^{9}\left(\mathrm{Nm}^{2}\right)$ and the structural damping ratio is 0.03 . The top tension is $T=6.8 \times 10^{7}(\mathrm{~N})$ and the flow velocity is $U=1.0 \mathrm{~m} / \mathrm{s}$.

\subsection{Effects of Top-End Periodic Motion}

In order to examine what will happen when top-end vessel sway is involved, we compared the riser dynamic responses for two cases, i.e. case 1: the riser is only suffering VIV, and case 2: the riser is suffering both VIV and vessel sway.

We calculated the displacement responses of the riser at different natural frequencies. Selected displacements, nondimensioned by the riser's outer diameter $D$ as $x / D$, are presented in Fig.3 where the top-end is swaying at the $6^{\text {th }}$ natural frequency of the riser. It is shown that the maximum RMS displacement (see Fig. 3b) for the riser experiencing both top-end sway and VIV is around 2.60, while the maximum RMS displacement (see Fig. 3a) is just 0.48 for the riser experiencing only VIV. So, we may say that top-end sway could introduce a larger riser response than the case without top-end motion.

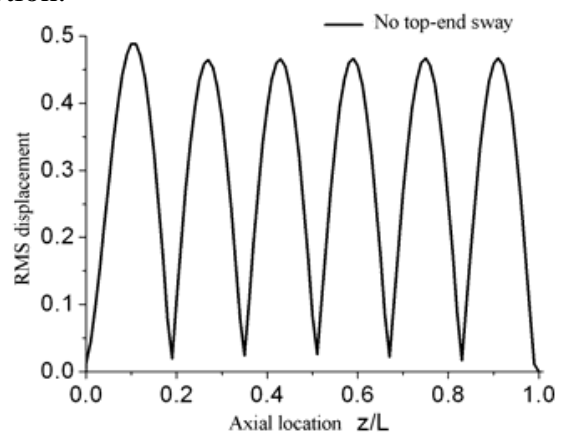

Figure 3a. The dynamic response without top end motion 


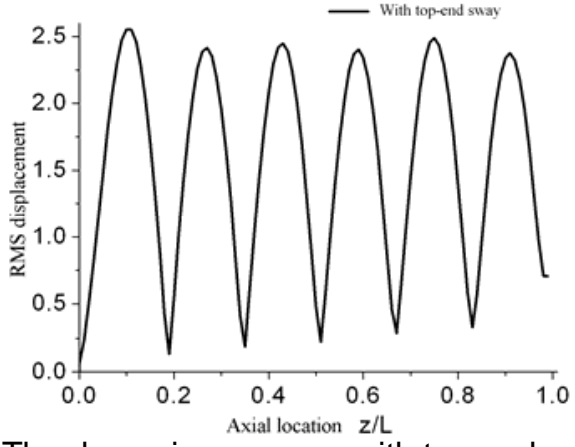

3b The dynamic response with top end motion Figure 3 Comparison between the dynamic response with and without top-end motion

Moreover, if comparing the displacement amplitude of the riser top end with the amplitudes at other locations along riser (see Fig. 4b), we note that the RMS displacement at the six peaks ranges from 2.30 to 2.60 , while the displacement at the top end $(\mathrm{z} / \mathrm{L}=1.0)$ is just 0.75 . In other words, the displacement amplitudes along riser span exceeds the original amplitude of the top-end. We call this phenomenon as response amplification. The amplification may be attributed to the moving boundary condition at riser top-end, of which the mechanism will be theoretically discussed.

To demonstrate the effects of moving boundary conditions on the riser dynamic response, we take an Euler beam as example, of which the governing equation can be written as

$$
E I \frac{\partial^{4} x(z, t)}{\partial z^{4}}-T \frac{\partial^{2} x(z, t)}{\partial z^{2}}+c \frac{\partial x(z, t)}{\partial t}+m \frac{\partial^{2} x(z, t)}{\partial t^{2}}=0
$$

One end of the beam is fixed, and another end is ongoing a sinusoidal vibration with the frequency $\omega_{0}$ and amplitude $B$. Thus the boundary conditions are

$$
\left\{\begin{array}{l}
x(L, t)=B e^{-i \omega_{0} t}, x^{\prime \prime}(L, t)=0 \\
x(0, t)=0, x^{\prime \prime}(0, t)=0
\end{array}\right.
$$

Assuming a solution, $x=e^{i(k x-\omega t)}$, of Eq. (5), we can get a dispersion equation, by substituting the assumed solution into Eq.(5), as follow

$$
E I k^{4}+T_{0} k^{2}-m \omega^{2}-i c \omega=0
$$

where $k$ is the wave number.

Then the response displacement can be written as

$$
x=\alpha_{1} e^{i\left(k_{1} x-\omega t\right)}+\beta_{1} e^{i\left(-k_{1} x-\omega t\right)}+\alpha_{2} e^{i\left(k_{2} x-\omega t\right)}+\beta_{2} e^{i\left(-k_{2} x-\omega t\right)}
$$

where $k_{1}$ and $k_{2}$ are the four roots of Eq.(7) as

$$
\begin{aligned}
& k_{1}=\sqrt{\frac{1}{2}\left(-\frac{T_{0}}{E I}+\sqrt{\left.\left(\frac{T_{0}}{E I}\right)^{2}+\frac{4\left(m \omega^{2}-i c \omega\right)}{E I}\right)}\right.} \\
& k_{2}=i \sqrt{\frac{1}{2}\left(\frac{T_{0}}{E I}+\sqrt{\left.\left(\frac{T_{0}}{E I}\right)^{2}+\frac{4\left(m \omega^{2}-i c \omega\right)}{E I}\right)}\right.}
\end{aligned}
$$

And the coefficients $\alpha_{1}, \alpha_{2}, \beta_{1}$ and $\beta_{2}$ are as follows:

$$
\begin{aligned}
& \alpha_{1}=\frac{-B k_{2}^{2} e^{-i k_{1} L}}{-k_{2}^{2} e^{-i k_{1} L}+k_{1}^{2} e^{-i k_{1} L}+k_{2}^{2} e^{i k_{1} L}-k_{1}^{2} e^{i k_{1} L}}, \\
& \alpha_{2}=\frac{B k_{1}^{2} e^{-i k_{2} L}}{-k_{2}^{2} e^{-i k_{2} L}+k_{2}^{2} e^{k_{2} L}+k_{1}^{2} e^{-i k_{2} L}-k_{1}^{2} e^{i k_{2} L},} \\
& \beta_{1}=\frac{B k_{2}^{2} e^{-i k_{1} L}}{-k_{2}^{2} e^{-i k_{1} L}+k_{1}^{2} e^{-i k_{1} L}+k_{2}^{2} e^{i k_{1} L}-k_{1}^{2} e^{i k_{1} L}}, \\
& \beta_{2}=\frac{-B k_{1}^{2} e^{i k_{2} L}}{-k_{2}^{2} e^{-i k_{2} L}+k_{2}^{2} e^{i k_{2} L}+k_{1}^{2} e^{-i k_{2} L}-k_{1}^{2} e^{i k_{2} L}}
\end{aligned}
$$

With above solutions, we can carry out dynamic response simulation of a beam suffering moving boundary condition. Fig. 4 presents the traces of the beam's response during a period of time at different frequencies, i.e. $\omega_{0}$ is respectively the beam natural frequencies $\omega_{5}$ and $\omega_{6}$ and a random frequency $\omega_{0}=1.94 \mathrm{~Hz}$. In Fig. 4 the left end is fixed while the right end is moving sinusoidally with an amplitude of $B=1 \mathrm{~m}$. It is seen that the displacements at the peaks along the beam are obviously larger than the displacements of the right end when the moving frequency of the boundary is consistent with the beam natural frequency, shown in both Fig. 4a and Fig. 4b. As mentioned above, we call it response amplification due to moving boundary condition.

Moreover, comparing the maximum displacements in Fig.4a with that in Fig. 4b, we note that the response amplification factor, $A / B$, gets larger when the mode order number is lower, e.g. the value of the amplification factor is 8.0 for mode 5 whereas 6.8 for mode 6 . If the frequency of the moving boundary is not consistent with the beam natural frequency (see Fig.4c), the response amplification, about 1.1, is not that pronounced.

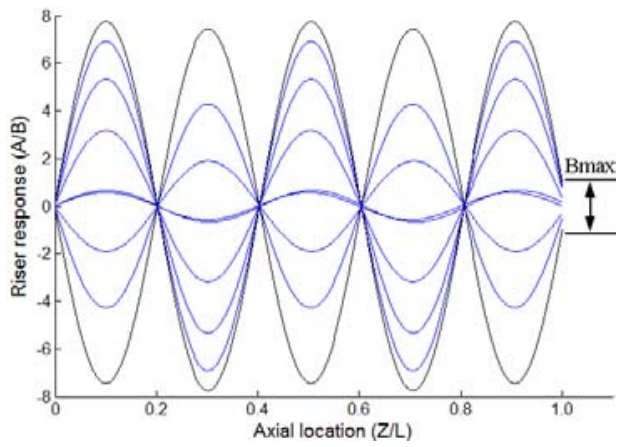

4a Modal trace of response at the frequency $\omega_{5}$ 


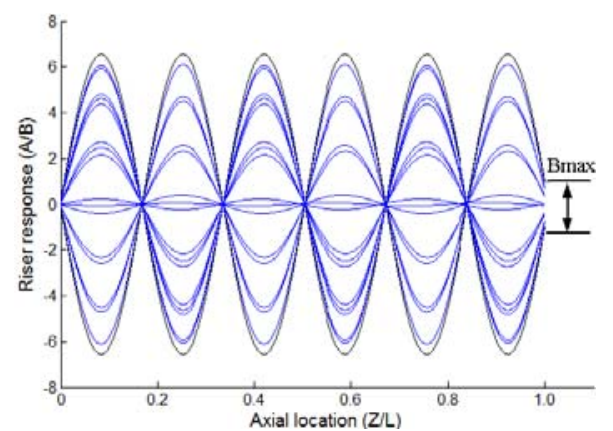

$4 b$

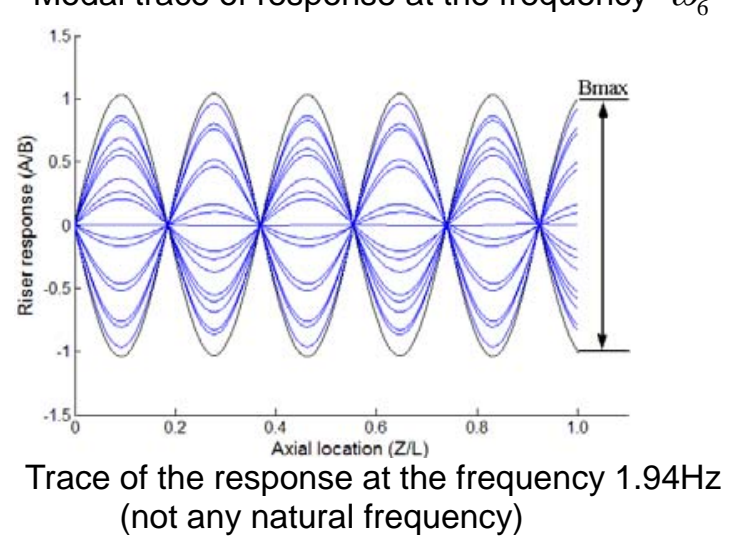

Figure 4. Trace of the response at different frequencies

\subsection{Effects of Sway Frequencies}

The riser dynamic responses are simulated while the topend is swaying with a $10 \mathrm{~m}$ amplitude. Both the sway cycle and the vortex-induced lift force cycle are same with the riser natural frequencies ranging from the $1^{\text {st }}$ to the $24^{\text {th }}$ Modes. Selected dynamic response displacements are presented in Fig. 5. Generally speaking, the displacements decrease as natural frequencies increasing. Or, the amplification factor drops with the increase of mode order number. It is also noted that the modal responses of lower-order modes are mostly dominated by standing wave, while the travelling wave can been seen obvioinusely in the responses of higher-order modes, see Fig. $5 \mathrm{~d} \sim 5 \mathrm{f}$ where there is no longer exact node. This is mainly because the damping of modes with higher order number becomes larger, and the modal dynamic response declines faster. Thus, the riser vibration introduced by top-end fluctuation may decline rapidly into a pretty little, even zero, value before it reaches the riser bottom end, then, to reflect backward. If there is just a pretty weak, or even no, reflecting wave to meet the approaching wave, it is harder to form a standing wave for modes with higher order number.

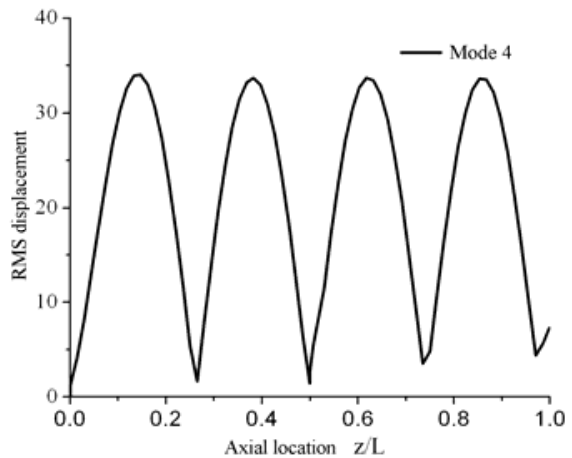

5a Displacement of mode 4

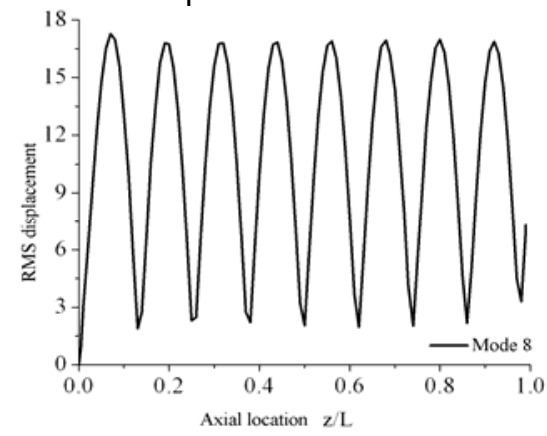

$5 \mathrm{~b} \quad$ Displacement of mode 8

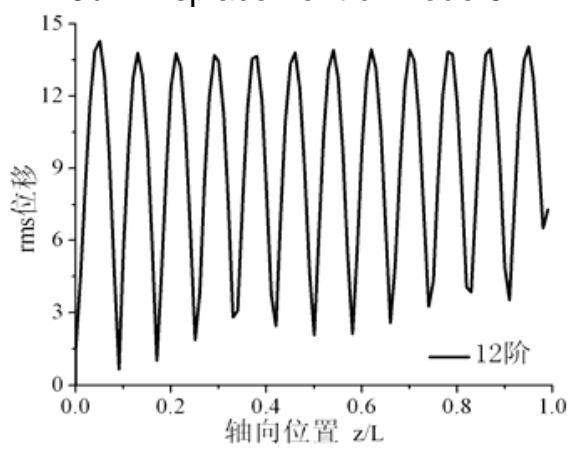

5c Displacement of mode 12

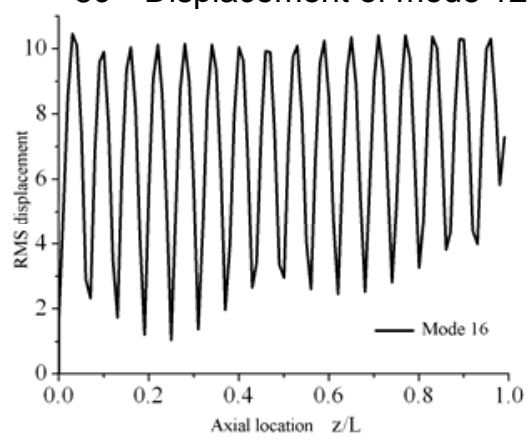

$5 d \quad$ Displacement of mode 18 


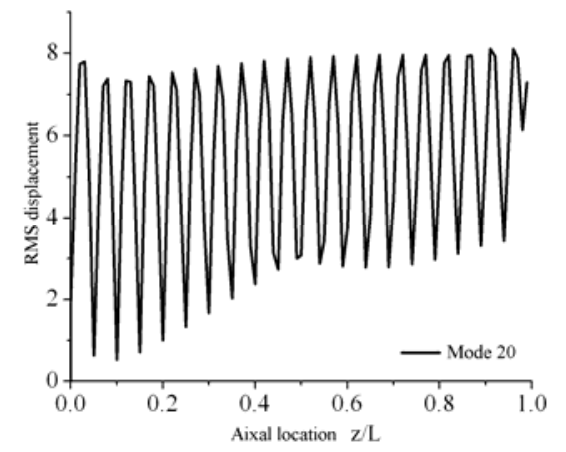

$5 e \quad$ Displacement of mode 22

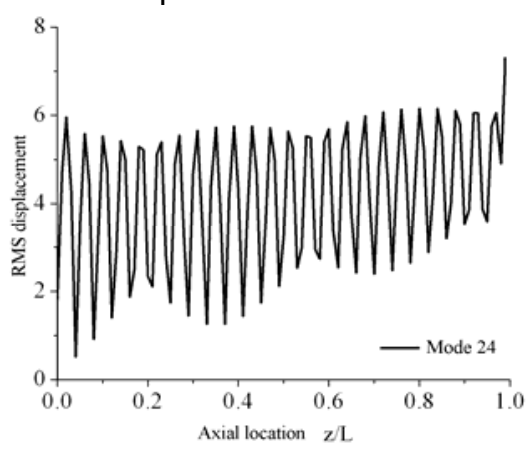

$5 f \quad$ Displacement of mode 24

Figure 5. Modal RMS displacement responses of riser undergoing VIV along with top end motion

\subsection{Effects of Sway Amplitudes}

Another factor which may influence riser dynamic response is sway amplitude. In fact, if normalized by the riser diameter $D$ as $2 \pi B / D$, the sway amplitude $B$ can be defined as the $K C$ number, $K C=2 \pi B / D$, which implies the relative motion between the top-end and water. The riser responses are simulated for several cases of the sway amplitude ranging from $B / D=1$ to 10 . Selected RMS displacements are shown in Fig. 6 where the vibrating frequency is at $\omega_{6}$, the $6^{\text {th }}$ natural frequency of the riser.

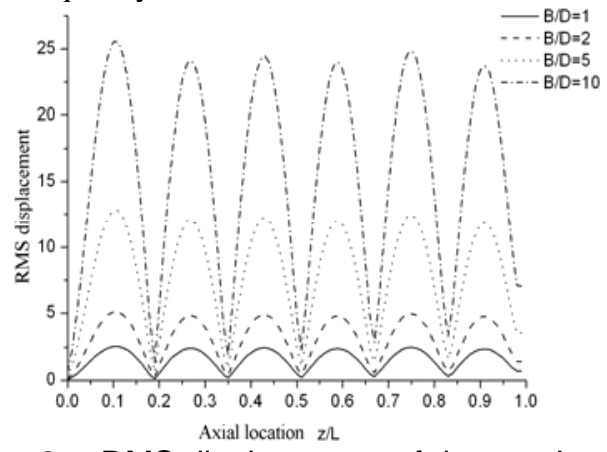

Figure 6. RMS displacement of riser undergoing different amplitudes of top motion

As shown in Fig.6, the riser displacement gets larger as the sway amplitude rising, e.g. the maximum RMS displacement increasing from 2.6 as $B / D=1$ to 16.5 as $B / D=10$. However, if we make a plot (shown in Fig. 7) of the nondimentional displacement $A / B$, a ratio of the riser amplitude to the top-end amplitude, versus the $K C$ number, the nondimentional amplitude $2 \pi B / D$, it is seen that the value of $A / B$ just slightly fluctuates around 2.5. Or, it changes little as the $K C$ number changes. We may say there is not profound influence of sway amplitude on the riser amplification factor.

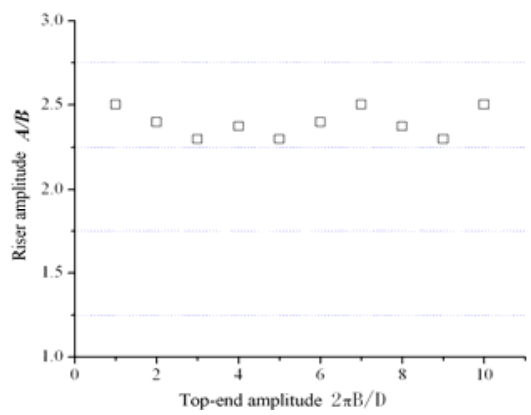

Figure 7. Effect of KC number on amplification factor

\section{CONCLUSIONS}

A coupled hydrodynamic force approach is developed based on the interaction between fluid and structural dynamics. The dynamic responses of the coupling system including both a floating top-end and a riser undergoing VIV are examined by means of finite element numerical simulations. Our results show that the riser displacement becomes several times larger than that of the riser without moving top-end. Another interesting phenomenon is that the top-end vibration may be amplified as propagating from the top to the bottom end along riser. Based on our numerical simulations, we draw following conclusions:

1)The response amplification gets more pronounced as the number of mode order falling. Generally speaking, the riser dynamic response, as well the response amplification, drops as vibrating frequencies rising. The dynamics responses of the modes with lower order number are mostly dominated by standing wave, while travelling wave can be observed on the responses of modes with higher order number.

2)Riser response displacement gets larger as sway amplitude rising. However, the value of nondimentional displacement $A / B$ changes little with top-end sway amplitude. Or, there is not remarkable effect of sway amplitude on response amplification factor.

In summary, for a coupling system of a top-end vessel together with submarine risers, the periodic motion of top-end vessel actually introduces a moving boundary condition and probably a consequent response amplification. This response amplification is critical for riser response, because that may directly cause large displacement and high stress somewhere along riser. It is always a serious concern for strength design of platform structure. Further, the relative motion of riser to amid fluid field changes the velocity distribution along riser, and that may introduce different vortex shedding modes and consequently new lock-in region. Therefore, a small top-end 
motion may turn out a large riser motion somewhere along rise span, perhaps causing significant VIV, which may not be anticipated from the small amplitude top-end motion.

On the other hand, moving top-end may cause a periodic riser motion. Then there is an unsteady relative motion between riser and fluid field. Actually, this introduces a new issue of unsteady VIV, which is quiet different from the steady VIV. Further studies are suggested on more challenging issues like unsteady VIV, experimental (or CFD) investigations on the lift force and structural motion and new prediction model.

\section{ACKNOWLEDGMENTS}

The authors of this paper would like to thank the financial supports provided by the National Natural Sciences Foundation (Grant No. 11232012 and 11372320).

\section{REFERENCES}

Bosman R.L.M., Hooker J., 1999. Elastic modulus characteristics of polyester mooring ropes. Proceedings of the Annual Offshore Technology Conference 1246-1251.

Chaplin, J.R., Bearman, P.W., Huera, F.J., et al. 2005. Laboratory measurements of vortex-induced vibrations of a vertical tension riser in a stepped current. Journal of Fluids and Structures 21, 3-24.

Chen W.M., Li M., Zheng Z.Q., 2012. Dynamic Characteristics and VIV of Deepwater Riser with Axially Varying Structural Properties. Ocean Engineering 42, 7-12.

Chen X.H., Ding Y., Zhang Y., 2006. Coupled dynamic analysis of a mini TLP: Comparison with measurements. Ocean Engineering 33, 93-117

Garrett D.L., 2005. Coupled analysis of floating production systems. Ocean Engineering 32, 802-816

Gopalkrishnan R., 1993. Vortex induced forces on oscillating bluff cylinders [Ph.D.Thesis]. MA, USA: MIT, Cambridge.

Govardhan R., Williamson C.H.K., 2004. Critical mass in vortex-induced vibration of a cylinder. European Journal of Mechanics B/Fluids 23, 17-27.

Heurtier J M, Buhan L, Fontaine E, 2001. Coupled dynamic response of moored FPSO with risers. Proceedings of the Eleventh International Offshore and Polar Engineering Conference 17-22.

Khalak, A,. Williamson, C.H.K., 1999. Motions, forces and mode transitions in vortex-induced vibrations at low massdamping. Journal of Fluids and Structures 13, 813-851.

Kim M.H., Arcandra T., Kim Y.B., 2001. Variability of spar motion analysis against various design methodologies /parameters. Proceedings of the ASME 20th International Conference on Ocean, Offshore and Arctic Engineering OMAE2001, June 2001, Rio de Janeiro, Brazil

Lee H.H., Wang P.W., 2000. Analytical solution on the surge motion of tension-leg twin platform structural systems. Ocean Engineering 27, 393-415.

Li B.B., Ou J.P., Teng B., 2010. Fully Coupled Effects of Hull, Mooring and Risers Model in Time Domain Based on An
Innovative Deep Draft Multi-Spar. China Ocean Engineering 24(2), 219-233.

Liao J.C., Vandiver J.M., 2002. Vortex-induced vibration of slender structure in unsteady flow. Ph.D. dissertation, Massachusetts Institute of Technology.

Ormberg H., Fylling I.J., Larsen K., Sodahl N., 1997. Coupled analysis of vessel motions and mooring and riser system dynamics. Proceedings of the ASME 16th International Conference on Ocean, Offshore and Arctic Engineering OMAE1997, April 1997, Yokohama, Japan

Pereira F.R., Gonçalves R.T., Pesce C.P. et al., 2013. A Model Scale Experimental Investigation on Vortex-Self Induced Vibrations (VSIV) of Catenary Risers, OMAE2013-10447. Proceedings of the ASME 32ndth International Conference on Ocean, Offshore and Arctic Engineering, June 9-14, Nantes, France.

Rampazzo F.P., Marcio M., Tsukamoto M.M. et al., 2008. Coupled Analysis of DP-Tugboat and Onshore PreAssembled SCR (Steel Catenary Riser) Transportation System, OMAE2008-57571, Proceedings of the ASME 27th International Conference on Ocean, Offshore and Arctic Engineering, June 15-20, 2008, Estoril, Portugal.

Sarpkaya T., 2004. A Critical review of the intrinsic nature of vortex-induced vibration. Journal of Fluids and Structures Mechanics 46, 389-447

Spanos P.D., Ghosh R., Finn L.D. et al., 2005. Coupled analysis of a spar structure: Monte Carlo and statistical linearization solutions. Journal of Offshore Mechanics and Arctic Engineering 127(1), 11-16.

Stansberg C.T., Ormberg H., Oritsl O., 2002. Challenges in deep water experiments: Hybrid approach. Journal of Offshore Mechanics and Arctic Engineering 124, 91-96

Tahara A., Kim M.H., 2003. Hull/mooring/riser coupled dynamic analysis and sensitivity study of a tanker-based FPSO. Applied Ocean Research 25, 367-382

Tahara A., Kim M.H., 2008. Coupled-dynamic analysis of floating structures with polyester mooring lines. Ocean Engineering 35(17-18), 1676-1685.

Trim, A.D., Braaten, H., Lie H. et al. 2005. Experimental investigation of vortex-induced vibration of long marine risers. Journal of Fluids and Structures 21, 335-361.

Vandiver J.K., 2002. A Universal Reduced Damping Parameter for Prediction of Vortex-Induced Vibration. Proceedings of the ASME 21st International Conference on Ocean, Offshore and Arctic Engineering OMAE2002, June 2002, Oslo, Norway

Wang D.Y., Ling G.C., 1998. Vortex-induced nonlinear vibration of TLP tethers under circumstances of platform oscillation. ACTA Oceannologica SINICA 20(5), 119-128. in Chinese

Wichers J.E.W., Voogt H.J., Roelofs H.W., Driessen P.C.M., 2001. DeepStar-CTR 4401 Benchmark Model Test. Technical Report No. 16417-1-OB, MARIN, Netherlands. 